\title{
Facial approximations: Characteristics of the Eye in a South African Sample
}

\author{
Heléne F. Dorfling ${ }^{\mathrm{a},{ }^{*}}$, Zarina Lockhat ${ }^{\mathrm{b}}$, Samantha Pretorius ${ }^{\mathrm{c}}$, Maryna Steyn ${ }^{\mathrm{d}}$, \\ Anna C. Oettlé a,e \\ ${ }^{a}$ Department of Anatomy, Faculty of Health Sciences, University of Pretoria, Pretoria, South Africa \\ ${ }^{\mathrm{b}}$ Department of Radiology, Faculty of Health Sciences, University of Pretoria, South Africa \\ ${ }^{\mathrm{c}}$ Department Actuarial Science, Faculty of Natural and Agricultural Sciences, University of Pretoria, South \\ Africa \\ ${ }^{\mathrm{d}}$ Human Variation and Identification Research Unit, School of Anatomical Sciences, Faculty of Health \\ Sciences, University of the Witwatersrand, South Africa \\ ${ }^{\mathrm{e}}$ Department of Anatomy and Histology, School of Medicine, Sefako Makgatho Health Sciences University, \\ Ga-Rankuwa, Pretoria, South Africa \\ * Corresponding author. \\ E-mail addresses: u27089691@tuks.co.za (H.F. Dorfling), zarina.lockhat@up.ac.za (Z. Lockhat), \\ Samantha.Pretorius@up.ac.za (S.Pretorius), maryna.steyn@wits.ac.za (M.Steyn), anna.oettle@smu.ac.za (A.C. \\ Oettlé)
}

\section{Abstract}

Although guidelines for facial approximations, including those for the eye, are in use in South Africa, limited data on African populations exist to confirm its validity. As precise placement of the eyes in facial approximations is of importance for facial recognition, this study tested established guidelines by measuring specific instrumental dimensions. Forty-nine cadavers from the Sefako Makgatho Health Sciences University and the University of Pretoria were dissected to determine the position of the canthi and the size and position of the eyeball in the orbit. Thirty cone beam computer tomography scans and 30 computer tomography scans from the Oral and Dental and Steve Biko Hospitals respectively were used to determine the size of the eyeball. Results from this study were compared to the published guidelines. The most prominent discrepancies included a more rectangular shape of the orbit, an oval shaped eyeball and a different position of the canthi. In African faces, the medial canthus was found to be located higher than the lateral canthus. The distance between the endocanthion and superior orbital margin was $17.7 \mathrm{~mm}$ and the exocanthion and superior orbital margin $19.5 \mathrm{~mm}$. Interpopulation differences may have an effect on facial approximations and its accuracy as is often demonstrated in practice. The findings of this study should be taken into consideration when designing population specific guidelines for reconstruction of the eye in South Africans of African ancestry.

\section{Key words:}

Facial approximations, Canthi, Eyeball, Orbit. 


\section{Highlights}

- Dimensions of the eye and orbit in South Africans varies from published guidelines

- A more rectangular orbit results in a more transversely elongated eyeball

- The eyeball is located $13.2 \mathrm{~mm}$ superiorly and $14.6 \mathrm{~mm}$ laterally from orbit centre

- The exocanthion is situated lower than the endocanthion

\section{Introduction}

Identification of unknown individuals is a challenge in the South African context. In cases where there is a strong suspicion regarding the identity of the unknown individual and a close relative is available, methods such as DNA comparison and dental record comparison are useful. However, because of socio-economic and other reasons in the South African context, unidentified individuals without known relationships are commonplace [1]. In these cases, it is not possible to identify unknown individuals with primary identifiers and therefore forensic facial reconstruction/approximation is often used to obtain information on a case $[1,2]$.

The facial reconstruction/approximation process always begins with the placement of the eyes. Facial recognition (especially of familiar individuals) is dependent on the morphology of the orbital area [3-7], therefore it is important to be precise and correct in placing the eyes [8] and associated features. The eyes are to be positioned supero-laterally in the orbit according to guidelines established by expert studies [8-15]. Although conflicting findings on the position of the eyes have been reported [16], several studies [8, 14, 15] provide strong evidence of a more superior and lateral placement of the eyeball in the orbit. Specific distances of this position have been established for some populations $[8,14]$, but it is uncertain how applicable these absolute values are in the South African context.

Variations in the position of the endocanthion and exocanthion are also reported in the literature. Although all researchers did not use directly comparable landmarks to define the position of the endocanthion and the exocanthion, the general trend indicates that the endocanthion is positioned lower than the exocanthion [8, 16]. A study by Stewart [17], however, found the endocanthion and exocanthion to be on the same level. It would therefore be of value to determine the position of these landmarks in South African groups.

Similarly, variations have been reported in the dimensions of the eyeball. Although the eyeball is often considered as almost spherical [15], slight elongation in certain axes has been reported in the literature $[15,18]$. The medio-lateral axis was found to often be longer than the superoinferior axis [18]. 
In South Africa, guidelines created for and based on other populations are often applied in facial approximations (Capt. T.M. Briers, personal communication, 2014). However, these guidelines may not necessarily be applicable in the South African context as a degree of interpopulation variation exists in facial features. It is postulated that these inter-population differences may have an effect on facial approximations and its accuracy, as is often demonstrated in practice. The less accurate a facial representation, the smaller the likelihood of an unknown individual being recognised and identified.

The purpose of this study was to assess specific features related to the eye in South Africans and compare it to established guidelines commonly used in the facial approximation process. The features assessed included the position of the eyeball within the orbit, the size of the eyeball and the position of the canthi.

\section{Materials \& Methods}

A total of 49 adult cadavers (38 males and 11 females, age range $22-73$ years, mean age 47 years) from the dissection halls of two South African universities, namely Sefako Makgatho Health Sciences University (SMU) and the University of Pretoria (UP), were used in this study. Bodies at UP generally had their origins from local hospitals in Pretoria [1], while those at SMU originated from a wider area of the Gauteng Province and some areas in the North West Province. Samples demonstrating damage, distortion, or any effects of desiccation due to embalming were excluded.

A total of 30 computer tomography (CT) scans (23 males, 7 females, age range 21 - 84 years, mean age 42 years) from Steve Biko Academic Hospital affiliated with UP and 30 cone beam computer tomography (CBCT) scans (17 males and 13 females, age range 18 - 64 years, mean age 33) from the Oral and Dental Hospital, UP, were also used for measurement and analyses. These hospitals service the greater Gauteng area, as well as parts of the Limpopo and North West provinces. Patients' heads were orientated in the standard natural head position for scanning - supine in the case of CT and sitting in the case of CBCT. The CT scans slices taken by a Siemens SOMATOM Sensation 64 scanner were $2 \mathrm{~mm}$ thick. CBCT scans were obtained using a Planmeca ProMax 3D scanner with a voxel size of $0.4 \mathrm{~mm}$. Scans were retrospectively analysed and excluded if not orientated in the desired plane, the implicated structures could not be clearly identified or injury to the orbital area was present. All cadavers and scans were of South Africans of African ancestry (hereafter referred to as South Africans (SA)). 
Ethics clearance was obtained from the Main Ethics and Research Committee, Faculty of Health Science, University of Pretoria (Cadaver sample: 8/2016; Scan sample: 183/2016) prior to commencement of this study. The Faculty of Health Sciences Research Ethics Committee complies with the SA National Act no. 61 of 2003 as it pertains to health research.

The orbital regions of 49 cadavers were dissected and measured to determine the position of the canthi, the position of the eyeball in the orbit and the diameters of the eyeball. A nonparametric Wilcoxon Rank Sum test (2-sided) was used to investigate whether differences occurred between sexes. As the $\mathrm{p}$ values for all individual measurements were non-significant $(p>0.05)$, male and female samples were pooled for the remainder of the analyses.

The position of the canthi was determined on cadavers by pinning the Frankfort Horizontal Plane (FHP) from porion to orbitale and marking a reference plane parallel to the FHP and tangent to the most superior point on the superior orbital margin (SOM). This is similar to the methodology followed by Stephan \& Davidson [8] and Stephan et al.[14]. The endo -and exocanthion were identified and pinned and four distances were measured namely (1) between the endocanthion and medial orbital margin (MOM) (en-MOM), (2) between the endocanthion and SOM (en-SOM), (3) between the exocanthion and lateral orbital margin (LOM) (ex-LOM) and (4) between the exocanthion and SOM (ex-SOM) (Fig. 1a).

To determine the position of the eyeball in the orbit of each cadaver, a circular cut was made approximately $5 \mathrm{~mm}$ outside of the orbital margin. The skin and orbicularis oculi muscle were removed, and the entire eyeball exposed by careful blunt dissection and removal of peri-orbital fat and tissue. Pins were placed perpendicular to the surface of the bone at the most extreme points on the LOM, MOM, IOM and SOM. Another set of four pins were placed at the shortest distances respectively from the LOM, MOM, IOM and SOM on the equator of the eyeball (an imaginary line encircling the globe of the eye equidistant from the anterior and posterior poles) [19]. Four distances were measured between the pins, namely (1) inferior equator to IOM (iIeqIOM), (2) superior equator to SOM (Seq-SOM), (3) lateral equator to LOM (Leq-LOM) and (4) medial equator to MOM (Meq-MOM) (Fig. 1b).

Two measurements were taken on the cadavers to determine the diameters of the eyeball, namely (1) medio-lateral diameter (distance between pins at medial and lateral equators i.e. Meq-Leq) and (2) supero-inferior diameter (distance between pins at superior and inferior equators i.e. Seq-Ieq) (Fig. 1c). Individual measurements (as shown in Fig. 1 b and c) were used to obtain the horizontal and vertical diameters of the orbit. For the horizontal diameter, 
the distances considered were Meq-MOM (Fig. 1b (4)); Meq-Leq (Fig. 1c (1)); and Leq-LOM (Fig. 1b (3)). To obtain the vertical diameter of the orbit, the distances added together were the Seq-SOM (Fig. 1b (2)); Seq-Ieq (Fig. 1c (2)); and Ieq-IOM (Fig. 1b (1))

CBCT and CT scans were imported into MevisLab [20] as Digital Imaging and Communications in Medicine (DICOM) files for measurements regarding the diameter of the eyeball. The ExaminerViewer function in MevisLab was used to visualise the 3D reconstruction of the files to ensure the correct voxel size and reconstruction. Region of Interest (ROI) Select was used to select a specific region of interest, enlarging the relevant areas, in this case the orbital area (Fig. 2). The OrthoView2D function was then used to visualise the region of interest and identify two points corresponding in all three planes (coronal, sagittal and transverse). The relevant points to determine the diameter of the eyeball were the most inferior, superior, medial, lateral, anterior and posterior points on the equator of the eyeball. Lastly, the function XMarkerListMaxDistance was used to measure the distance between the identified points. Scans were orientated, points identified and measurements taken on a multiplanar level as the relevant landmarks and distances were not necessarily visible on a single plane simultaneously. The points, however, retained their respective three-dimensional (3D) positions regardless of scrolling through the slices. The dimensions reflecting the size and shape of the eyeball included the antero-posterior diameter (Aeq-Peq) (Fig. 3a), the mediolateral diameter (Meq-Leq) (Fig. 3b) and the supero-inferior diameter (Seq-Ieq) (Fig. 3c). Although visualisation of the eyeball on the 2D figure is not that clear, by scrolling up and down on the 3D image, the borders of the eyeball could be more readily identified thus enabling measurements. Non-parametric Wilcoxon Rank Sum tests (2-sided) once again determined non-significant variations $(\mathrm{p}>0.05)$ between male and female, thus samples were pooled. 


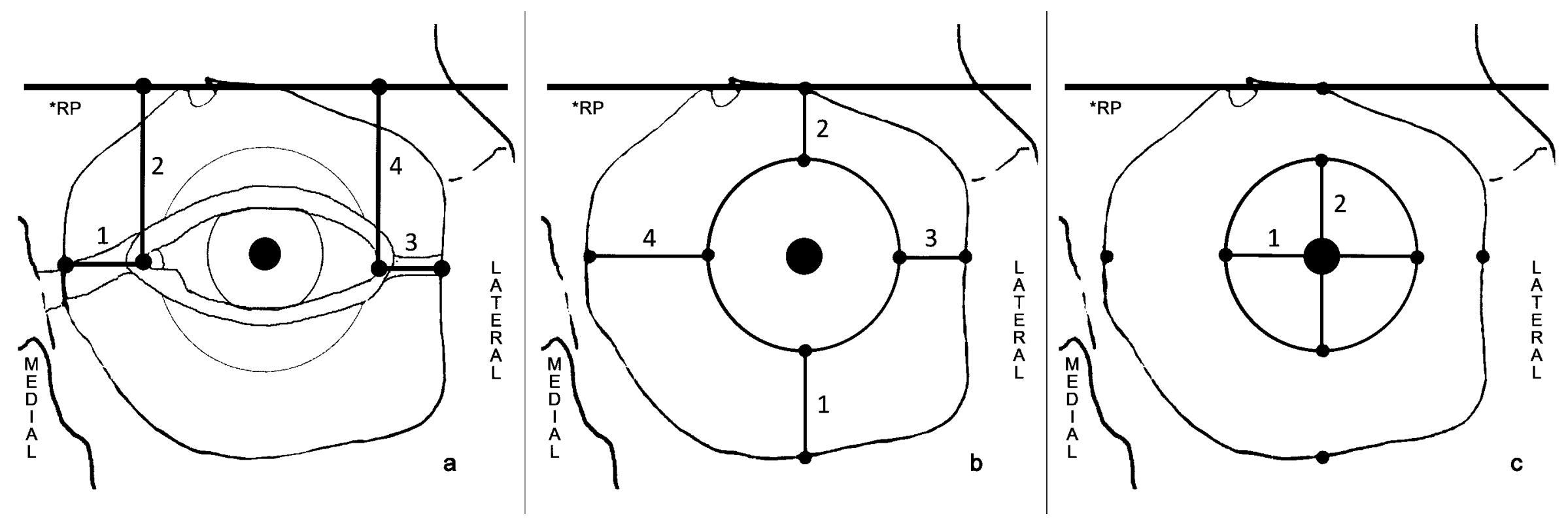

FIG. 1. Orbital and optic measurements. *RP: Reference plane parallel to FHP

a) Position of the canthi:

1: distance between the medial canthus and MOM

2: distance between the medial canthus and SOM reference plane

3: distance between the lateral canthus and LOM

4: distance between the lateral canthus and the SOM reference plane b) Position of the eyeball in the orbit

1: distance between the inferior equator and the IOM

2: distance between the superior equator and SOM

3: distance between the lateral equator and LOM

4: distance between the medial equator and MOM c) Size of the eyeball

1: medio-lateral diameter (distance from the medial equator to the lateral equator)

2: supero-inferior diameter (distance from the superior to inferior equator) 


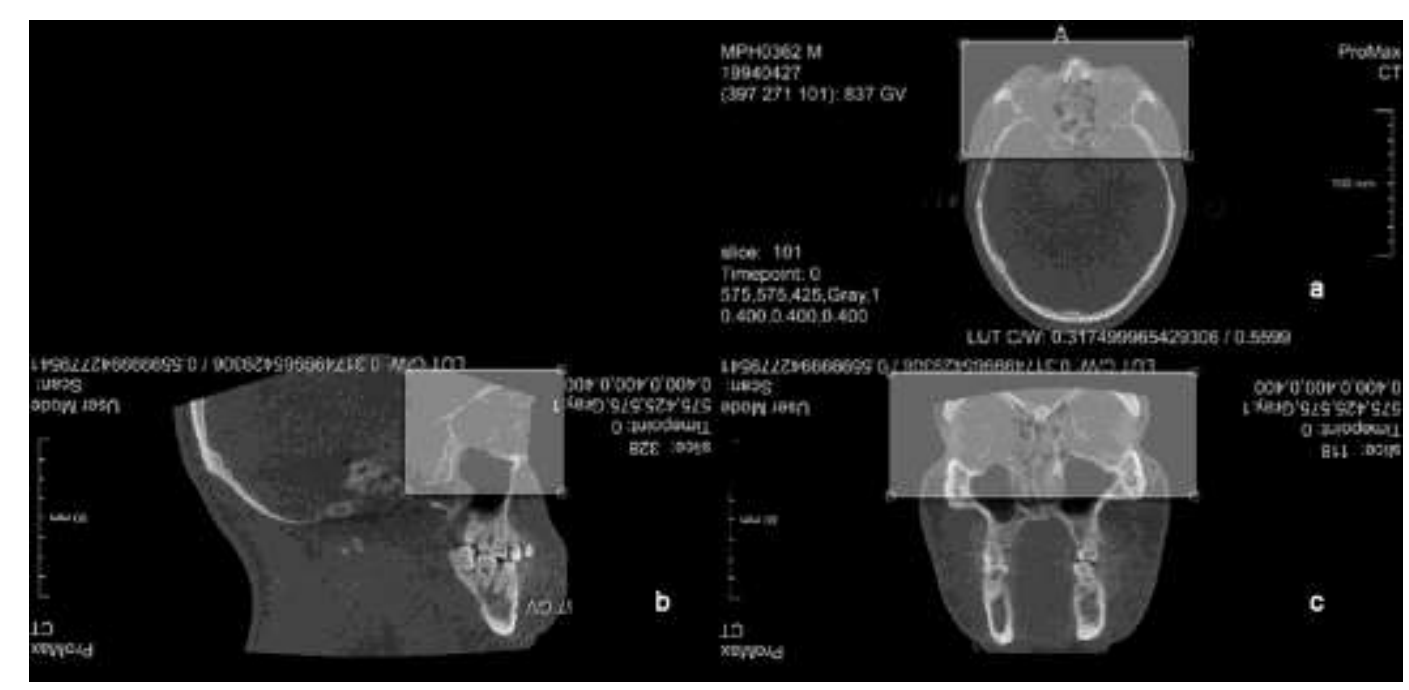

FIG. 2. Selecting the region of interest on MevisLab.

a) Transvers plane; b) Sagittal plane and c) Coronal plane
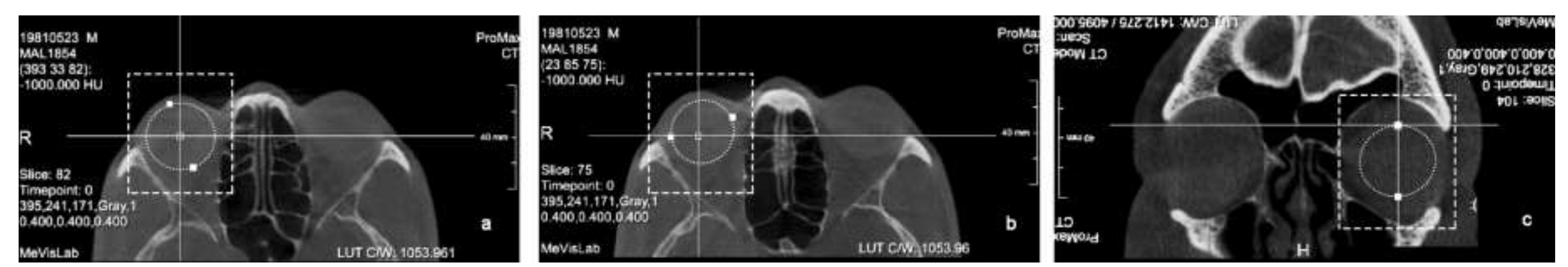

FIG. 3. Measuring the diameters of the eyeball between white squares.

a) Antero-posterior diameter; b) Medio-lateral diameter; c) Supero-inferior diameter 
The data were tested for variations between the sexes by means of a non-parametric Wilcoxon Rank Sum test (2-sided). Male and female samples were pooled together as there were no statistical differences between the sexes (except CBCT Meq-Leq where $\mathrm{p}<0.05$ ).

Comparisons were conducted between the measurements of the eyeball for all three modalities (dissection, CT and CBCT) utilising the Kruskal-Wallis test. The Kruskal-Wallis test is a nonparametric version of the classical one-way ANOVA, and an extension of the Wilcoxon Rank Sum test to more than two groups. Further comparisons on the medio-lateral and superoinferior diameters were done between two modalities at a time by means of the non-parametric Wilcoxon Rank Sum test, i.e. CT vs CBCT; CT vs dissection and dissections vs CBCT.

Intra-observer repeatability was assured for all measurements by obtaining three measurements for each dimension and calculating the technical error of measurement (TEM). Inter-observer repeatability testing was performed by obtaining measurements from one other observer. A total of 38 cadavers as well as all CT and CBCT scans were re-measured for all parameters. Interclass Correlation Coefficient A-1 (ICC) testing was done to compare measurements obtained by the two different observers.

\section{Results}

TEM results for cadaver measurements were all very low, with the mean reported as $<0.02$ $\mathrm{mm}$. CT scan TEM results were all calculated as $<0.2 \mathrm{~mm}$. CBCT scan results however, were less accurate with TEM results for Meq-Leq calculated at $0.88 \mathrm{~mm}$, Seq-Ieq at $0.81 \mathrm{~mm}$ and Aeq-Peq at $1.31 \mathrm{~mm}$. The accuracy of the measurements, when repeated by the same researcher, was considered higher the closer the TEM was to zero. Therefore, due to such small TEM's, the average measurement for each parameter was used for all further statistical analyses.

Descriptive statistics of the dimensions describing specific features of the eye including the position of the canthi, the position of the eyeball and the size of the eyeball were calculated and are summarised in Figures 4, 5 and 6 respectively.

Regarding the position of the canthi (Fig. 4), the endocanthion was found to be located higher and closer to the orbital margin than the exocanthion. Distances between ex-SOM (4) were found to be greater than the en-SOM $(2)(p<0.01)$, indicating that the exocanthion is located lower than the endocanthion in relation to the SOM reference plane. 
From Fig. 5 it can be noted that the eyeball is positioned supero-laterally within the orbit. A statistically significant difference between the distances of the Seq-SOM and Ieq-IOM ( $<$ < 0.01 ) and the Meq-MOM and Leq-LOM ( $<$ <0.01) was found. Fig. 5 illustrates that the distances between Seq-SOM (2) and Leq-LOM (3) were smaller than the distances between Ieq-IOM (1) and Meq-MOM (4), indicating that the eyeball is located more supero-laterally within the orbit. The dimensions of the eyeball with all modalities demonstrated a transverse elongation. The diameter of the eyeball (Fig. 6) as measured on cadavers (1), indicated that the medio-lateral diameter was greater at statistically significantly levels than the supero-inferior diameter $(\mathrm{p}<0.01)$. Similar results were found with CT $(2)(\mathrm{p}<0.01)$ and CBCT $(3)$

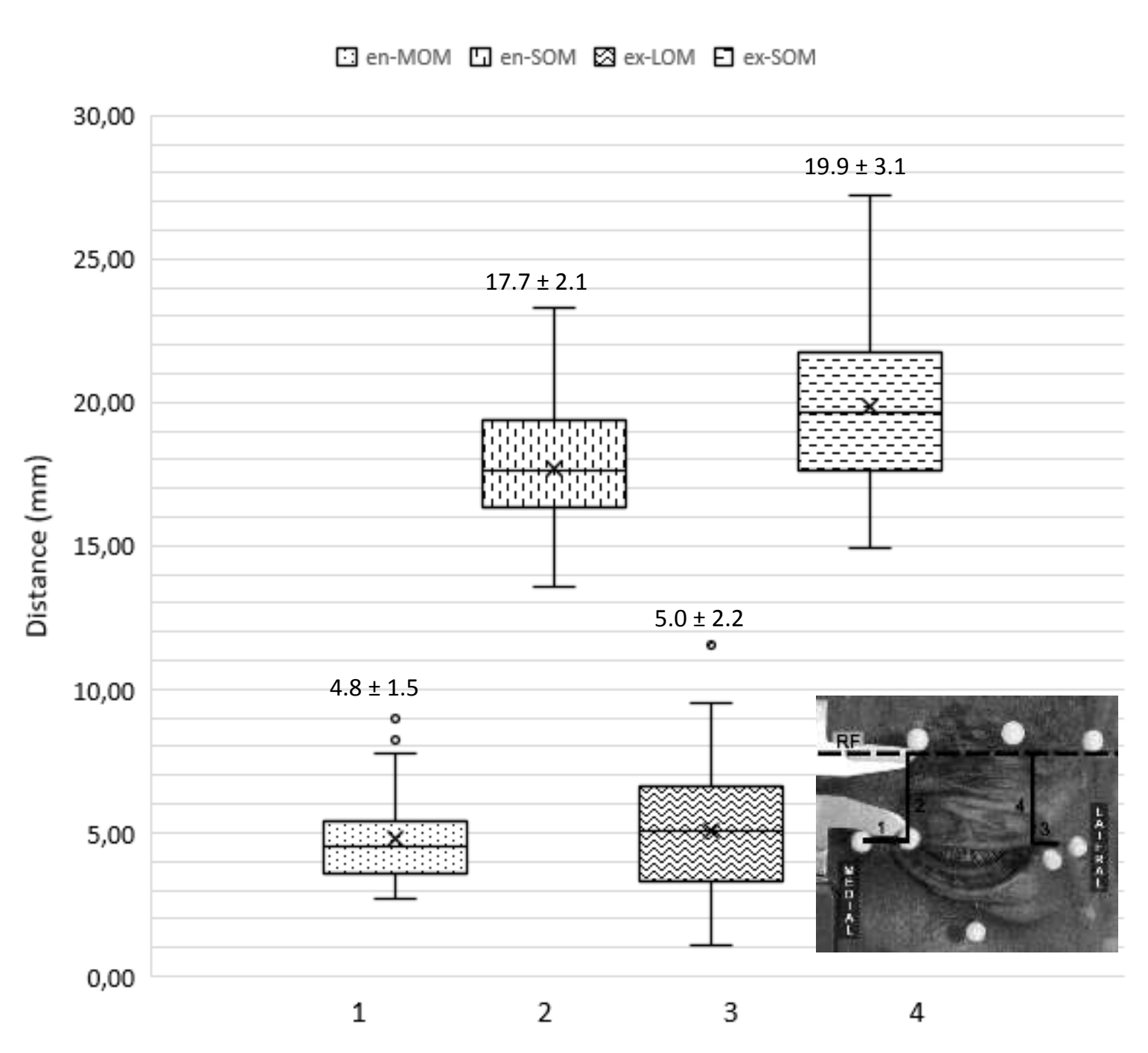

FIG. 4. Basic descriptive statistics for the measurements pertaining to the position of the canthi. (1) Distance between endocanthion and MOM, (2) distance between endocanthion and SOM, (3) distance between exocanthion and LOM and (4) distances between exocanthion and $S O M$ 


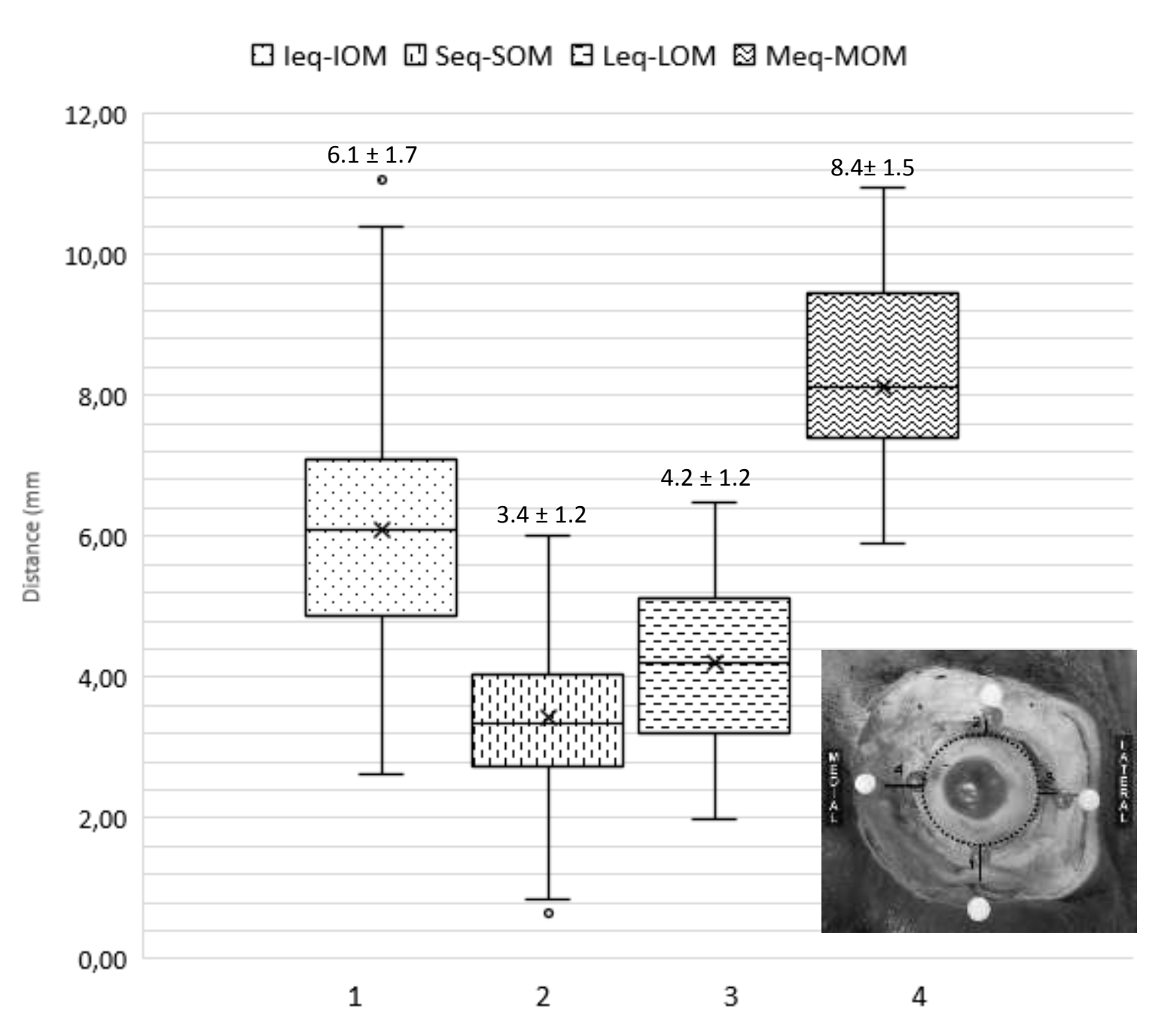

FIG 5. Basic descriptive statistics for measurements pertaining to the position of the eyeball in the orbit in dissections. (1) Distances between the inferior equator and the IOM, (2) distances between the superior equator and the SOM, (3) distances between the lateral equator and the LOM and (4) distances between the medial equator and the MOM 


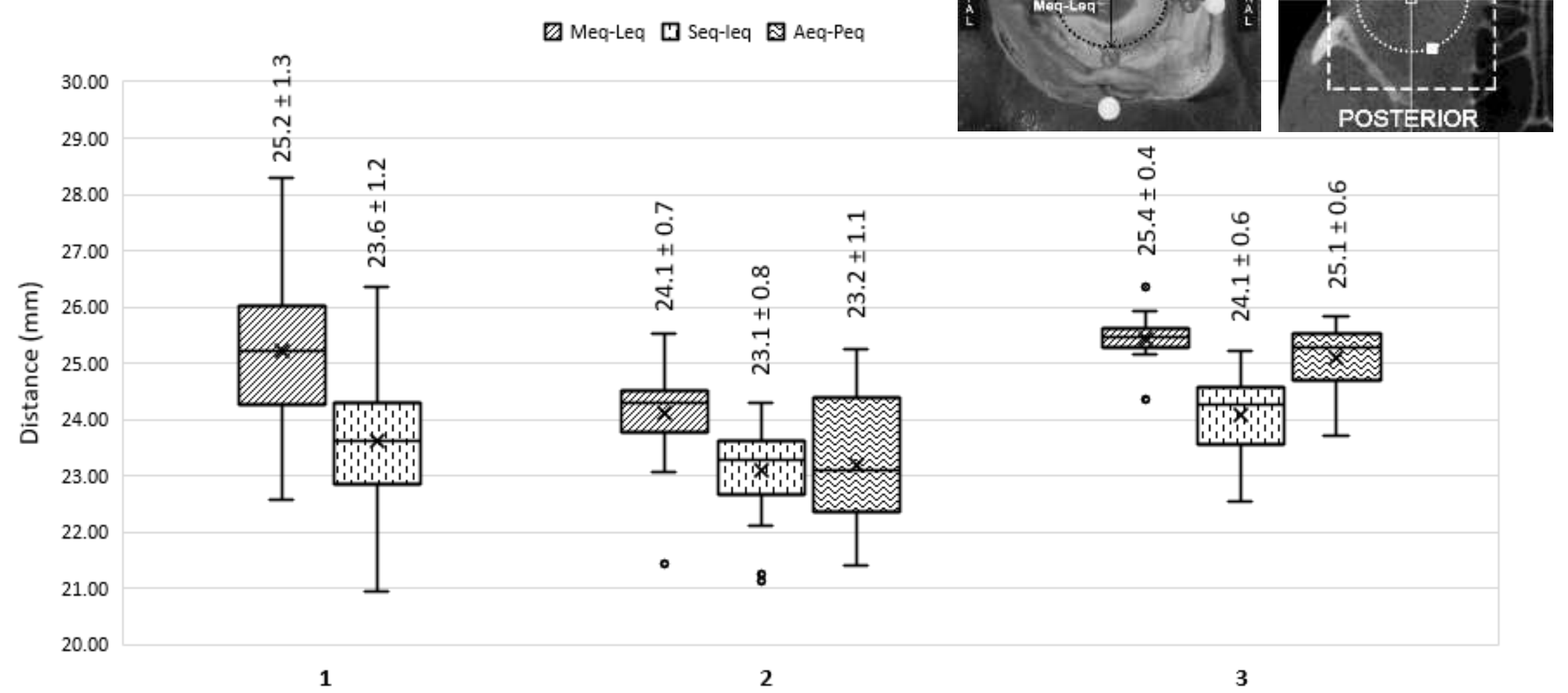

FIG. 6. Basic descriptive statistics for measurements pertaining to the size of the eyeball in all three modalities: (1) Dissections, (2) CT scans and (3) CBCT scans.

Kruskal-Wallis test (dissections vs CT vs CBCT) for medio-lateral diameter p-value $<0.01$

Kruskal-Wallis test (dissections vs CT vs CBCT) for supero-inferior diameter p-value $<0.01$

Wilcoxon Rank Sum test (CT vs CBCT) for antero-posterior diameter p-value $<0.01$ 
Calculations show that the width of the orbit is consistently greater that the height of the orbit, $(p<0.01)$ indicating a more rectangular shaped orbit. The shape of the eyeball thus reflects the shape of the orbits.

\section{Statistical comparisons between modalities}

Comparisons between two modalities were performed by utilising a Wilcoxon Rank Sum test for each of the three diameters (medio-lateral, supero-inferior and antero-posterior) of the eyeball. Box-and-whisker plots illustrate the variations in measurements obtained by using the different modalities (Fig. 6).

The dissection and CBCT measurements of the medio-lateral and supero-inferior dimensions of the eyeball showed a greater agreement than did CT measurements vs. CBCT measurements. This is demonstrated in the non-statistically significant differences demonstrated in the CBCT vs dissection derived means $(\mathrm{p}=0.39)$, while the variation between $\mathrm{CT}$ vs dissection measurements concerning the medio-lateral diameter was statistically significant $(\mathrm{p}<0.01)$ and so was CBCT vs CT $(\mathrm{p}<0.01)$. When considering the supero-inferior diameter, a statistically significant difference existed when comparing CBCT and CT ( $\mathrm{p}<0.01)$; however, this difference was less significant when comparing CBCT to cadaver measurements $(p=0.02)$. The variation in the supero-inferior diameters when comparing CT to dissection measurements was not statistically significant $(\mathrm{p}=0.05)$. CBCT data had the highest mean values, followed by the dissection data and then the CT data. Dissection data, however, had the greatest range, followed by CT data and lastly CBCT data. When comparing CBCT to CT measurements for the antero-posterior diameter, a statistically significant difference was observed $(\mathrm{p}<0.01)$.

Table 1 summarises the descriptive relationships for measurements performed on dissections, CBCT and CT scans. From the relationship between measurements, it can be seen that the distance from the exocanthion to SOM was greater than the distance from the endocanthion to the SOM, indicating that the exocanthion was on average located lower than the endocanthion. The width of the orbit was greater than the height, indicating that the orbit was more rectangular shaped. It can also be seen that the distance of the medial equator to the MOM and the distance of the inferior equator to the IOM was greater than the distance between the lateral equator to LOM and superior equator to SOM, demonstrating a more supero-laterally positioned eyeball. 
Table 1. Descriptive relationships between measurements

\begin{tabular}{|c|c|c|c|c|c|c|c|c|}
\hline \multirow[b]{2}{*}{ Measurements compared } & \multirow[b]{2}{*}{ Description } & \multirow[b]{2}{*}{ Result } & \multicolumn{2}{|c|}{$\begin{array}{l}\text { Dissections (Wilcoxon } \\
\text { Rank Sum Test) }\end{array}$} & \multicolumn{2}{|c|}{$\begin{array}{l}\text { CBCT } \\
\text { (Signed Rank Test) }\end{array}$} & \multicolumn{2}{|c|}{$\begin{array}{l}\text { CT } \\
\text { (Signed Rank Test) }\end{array}$} \\
\hline & & & Ratio & p-value & Ratio & p-value & Ratio & p-value \\
\hline en-SOM vs ex-SOM & $\begin{array}{l}\text { Vertical position } \\
\text { of the canthi }\end{array}$ & $\begin{array}{l}\text { ex-SOM > } \\
\text { en-SOM }\end{array}$ & 1.12 & $<0.01$ & & & & \\
\hline $\begin{array}{l}\text { Width of orbit vs } \\
\text { Height of orbit }\end{array}$ & Orbital shape & $\begin{array}{l}\text { Width > } \\
\text { Height }\end{array}$ & 1.14 & $<0.01$ & & & & \\
\hline $\begin{array}{l}\text { Meq - MOM vs Leq - } \\
\text { LOM }\end{array}$ & $\begin{array}{l}\text { Horizontal } \\
\text { position of } \\
\text { eyeball in the } \\
\text { orbit }\end{array}$ & $\begin{array}{l}\text { Meq - } \\
\text { MOM > Leq } \\
- \text { LOM }\end{array}$ & 1.98 & $<0.01$ & & & & \\
\hline $\begin{array}{l}\text { Seq - SOM vs Ieq - } \\
\text { IOM }\end{array}$ & $\begin{array}{l}\text { Vertical position } \\
\text { of eyeball in the } \\
\text { orbit }\end{array}$ & $\begin{array}{l}\text { Ieq }-\mathrm{IOM}> \\
\text { Seq }-\mathrm{SOM}\end{array}$ & 1.79 & $<0.01$ & & & & \\
\hline $\begin{array}{l}\text { Medial - Lateral } \\
\text { equators vs } \\
\text { Superior - Inferior } \\
\text { equators }\end{array}$ & Shape of eyeball & $\begin{array}{l}\text { Meq-Leq > } \\
\text { Seq-Ieq }\end{array}$ & 1.07 & $<0.01$ & 1.05 & $<0.01$ & 1.04 & $<0.01$ \\
\hline
\end{tabular}


The medio-lateral diameter was also greater than the supero-inferior diameter, indicating that the eyeball is elongated or oval shaped in the transverse axis.

ICC tests for cadaver measurements performed consistently, with excellent agreement (ICC > 0.90) between observers for ex-SOM, Meq-MOM, Leq-LOM, Ieq-IOM, and Leq-Meq. Good agreement (ICC > 0.85) was found between observers for en-SOM, Seq-SOM, Seq-Ieq, however the ex-LOM showed only moderate agreement $(\mathrm{ICC}=0.68)$ and the en-MOM dimension displayed poor inter-observer repeatability $(\mathrm{ICC}=0.04)$. Inter-observer repeatability tests for all CT and CBCT scan measurements were reported as less than 0.21 .

\section{Discussion and conclusions}

In this study, specific dimensions (absolute measurements and relationships between measurements) of the eye and orbit in South Africans were determined. Figure 7 summarises the findings recorded in the literature and mean dimensions observed in this study. Integration of the measurements obtained from the three modalities used (cadaver dissections, CT and CBCT) demonstrates that the exocanthion was positioned lower than the endocanthion, the orbit was rectangular-shaped and the oval shaped eyeball was situated in the superolateral aspect of the orbit. Findings regarding the shape of the orbit were in agreement with Krogman (1955) and others stating that the orbits of skulls of Africans are more rectangular than those from other populations.
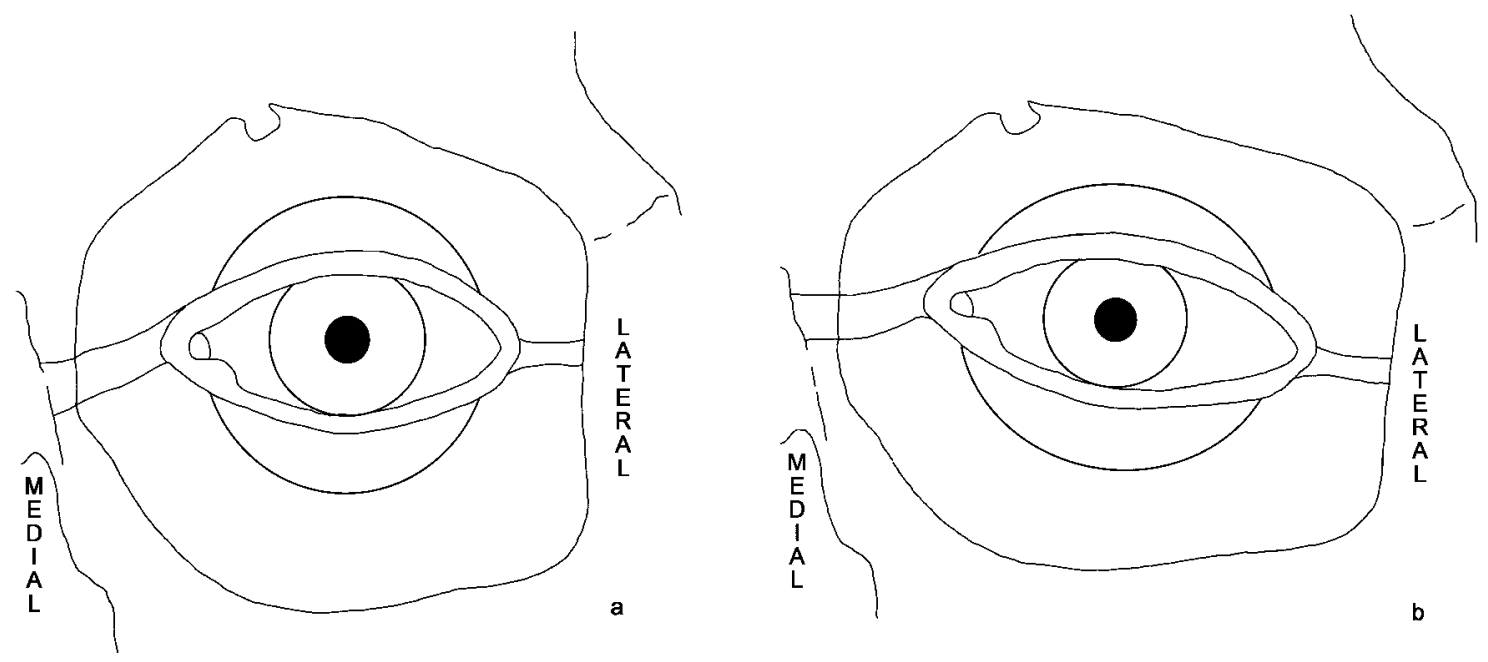

FIG 7. Graphic illustration of a) the expected findings and $b$ ) the mean dimensions of this study sample 
While most of the cadaver measurements had good inter-observer repeatability, the en-MOM dimension displayed poorly ( $\mathrm{ICC}=0.04$ ). This observation might be explained by the exact placement of the MOM that is not clearly defined. Unlike the lateral orbital margin, the MOM is less well defined, rounded and irregular. Inter-observer repeatability tests on CBCT and CT scan measurements had a similar performance but were less well than expected (All ICC < 0.21) as compared to intra-observer tests. Mean values of most measurements, however, differed with less than $2 \mathrm{~mm}$ in general, which may be considered acceptable [21].

Variations in the exact position of the endocanthion and exocanthion are reported in the literature. In most research done previously, the endocanthion was reported to be situated lower than the exocanthion. According to Stephan and Davidson [8], the endocanthion in an Australian population lies lower than the exocanthion, with the endocanthion approximately $19.5 \mathrm{~mm}$ below the SOM reference plane and the exocanthion $18.5 \mathrm{~mm}$ below the SOM reference plane. Kim et al. [16] similarly reported that in their sample of Korean individuals, the endocanthion is found lower than the exocanthion, at $22.8 \mathrm{~mm}$ and $20.2 \mathrm{~mm}$ respectively below the SOM. However, although specific distances are quite similar, especially to [8], in contrast to these studies reporting on the position of the canthi, the current study found the endocanthion to be positioned higher than the exocanthion. The endocanthion was located 17.7 $\mathrm{mm}$ below the SOM reference plane and the exocanthion was situated $19.5 \mathrm{~mm}$ below the SOM reference plane. It is reasonable to postulate that the differences in position observed in this population may be related to the population specific variation in the morphology of the zygomatic bone as well as the zygomatic processes of the frontal and maxillary bones contributing to the margins of the orbit resulting in variations in the shape of the orbital border [22].

Reports regarding the distance of the exocanthion from the lateral orbital margin are remarkably similar at $4.5 \mathrm{~mm}$ [8], $4.7 \mathrm{~mm} \mathrm{[16]} \mathrm{and} 5 \mathrm{~mm}$ medial to the malar tubercle [23], and are comparable to our findings $(5.0 \mathrm{~mm})$. The distance from the medial orbital margin to the endocanthion is more variable: $4.8 \mathrm{~mm}$ [8], $9.8 \mathrm{~mm}$ [16], approximately $2 \mathrm{~mm}$ lateral to the MOM [23] and $4.8 \mathrm{~mm}$ (current study). The variation noted in the Korean sample [16] might be due to the presence of epicanthal folds in people of Asian descent. Epicanthal folds that cover the endocanthion may influence the inclination of the eye fissure's in the longitudinal axis, by shifting the medial point of the axis from the endocanthion to a lower positioned point at the crossing of the epicanthus with the rim of the lower eyelid [24]. It may also be influenced by the size and shape of the nasal root [24]. 
Considering the position of the eyeball in the orbit, measurements found in this study are consistent with many previous studies, indicating a more supero-laterally placed eyeball [8-11, 13, 14]. Distances observed in this population group were measured as $3.4 \mathrm{~mm}$ from the SOM, $6.1 \mathrm{~mm}$ from the IOM, $8.3 \mathrm{~mm}$ from the MOM and $4.2 \mathrm{~mm}$ from the LOM. Distances reported by other authors $[8,9,11,14]$ ranged between $4.0-5.0 \mathrm{~mm}$ from the SOM; $6.8-7.8 \mathrm{~mm}$ from the IOM; $6.5-8.0 \mathrm{~mm}$ from the MOM and $3.9-4.5 \mathrm{~mm}$ from the LOM. Although some measurements obtained in this study are similar to the other studies (e.g., $[8,9,11,14])$, small differences are observed cumulatively in the transverse axis (i.e. the medial equator to MOM plus lateral equator to LOM), as compared to the longitudinal axis (i.e. the superior equator to SOM plus inferior equator to IOM). This is indicative of a greater periorbital space transversely compared to a smaller periorbital space observed longitudinally. These greater distances in the transverse axis in the African group is probably related to the more rectangular shape of the orbit in this group.

Evaluation of the diameters of the eyeball (summarised in Table 2) indicate an oval shape (elongation in the transverse axis) which has also been observed by clinicians $[18,25]$. The medio-lateral diameter of the eye in Africans is slightly greater than the supero-inferior diameter, but also to a small extent (approximately $1 \mathrm{~mm}$ ) greater than in other population groups $[15,18]$. All diameters were marginally greater on CBCT than reported on other modalities and other groups while the CT findings were more in agreement with previous findings $[15,18,25,26]$.

In conclusion, it was found that dimensions of the eye itself and its relative position in the orbit in South Africans varied minimally from the established guidelines. However, the more rectangular orbit resulted in a more transversely elongated eyeball which was located superolaterally within the orbit. The exocanthion in this group was situated lower than the endocanthion, in contrast to what was found in other studies. These variations can have a significant impact on the approximation of this pivotal feature. The combined effect of these variations can influence the likelihood of an unknown individual being identified, and therefore population specific standards should be used in cases of facial approximation. 
Table 2. Summary of variations in eyeball diameters

\begin{tabular}{|c|c|c|c|c|c|c|c|c|c|c|}
\hline Author(s) & Year & Modality & $\mathbf{n}$ & Ancestry & $\begin{array}{l}\text { Antero- } \\
\text { posterior } \\
\text { diameter }\end{array}$ & SD & $\begin{array}{l}\text { Medio- } \\
\text { lateral } \\
\text { diameter }\end{array}$ & SD & $\begin{array}{l}\text { Supero- } \\
\text { inferior } \\
\text { diameter }\end{array}$ & SD \\
\hline $\begin{array}{l}\text { Wilkinson \& } \\
\text { Mautner }\end{array}$ & 2003 & MRI & 39 & European & 23.28 & 1.66 & - & - & - & - \\
\hline $\begin{array}{l}\text { Guyomarc'h } \\
\text { et al. }\end{array}$ & 2012 & $\mathrm{CT}$ & 375 & French & 23.7 & - & 24.3 & - & 24.6 & - \\
\hline $\begin{array}{l}\text { Bekerman et } \\
\text { al. }\end{array}$ & 2014 & $\mathrm{CT}$ & 500 & $\begin{array}{l}\text { Mixed } \\
\text { ancestral } \\
\text { groups }\end{array}$ & $\begin{array}{l}22.1 \quad- \\
24.9\end{array}$ & - & $\begin{array}{l}24.1- \\
24.3\end{array}$ & - & $\begin{array}{l}23.7 \quad- \\
23.8\end{array}$ & - \\
\hline Özer et al. & 2016 & $\mathrm{CT}$ & 198 & Turkish & $\begin{array}{l}22.7 \\
\text { (females) } \\
23.3 \\
\text { (males) }\end{array}$ & $\begin{array}{l}6.38 \\
\text { (females) } \\
0.88 \\
\text { (males) }\end{array}$ & - & - & - & - \\
\hline This study & 2017 & $\begin{array}{l}\text { Dissectio } \\
\text { ns }\end{array}$ & 36 & SA & - & - & 25.2 & 1.42 & 23.6 & 1.29 \\
\hline This study & 2017 & $\mathrm{CT}$ & 30 & SA & 23.2 & 1.07 & 24.1 & 0.73 & 23.1 & 0.75 \\
\hline This study & 2017 & CBCT & 30 & SA & 25.1 & 0.56 & 25.4 & 0.38 & 24.1 & 0.64 \\
\hline
\end{tabular}




\section{Acknowledgements}

The authors would like to thank Dr. A. Uys for the use of CBCT scans. We are also indebted

to Mr. H. Human (Sefako Makgatho Health Sciences University) and Prof. M.C. Bosman

(University of Pretoria) for permission to use the cadaver collection for dissections. Funding for this research was provided by AESOP and the Rescom of the Faculty of Health Sciences at the University of Pretoria. The research of M Steyn is supported by the National Research Foundation (NRF) of South Africa. Any opinions, findings and conclusions or recommenddations expressed in this study are those of the author and therefore the NRF does not accept any liability in regard thereto.

\section{References}

[1] E.N. L'Abbé, M. Loots, J.H. Meiring, The Pretoria Bone Collection: a modern South African skeletal sample, HOMO - Journal of Comparative Human Biology 56 (2005) 197-205. [2] D. Cavanagh, M. Steyn, Facial reconstruction: soft tissue thickness values for South African black females, Forensic Science International 206 (2011) 215. e1-215. e7.

[3] E. Hjelmas, J. Wroldsen, Recognizing faces from the eyes only, In Proceedings of the 11th Scandinavian Conference on Image Analysis, Citeseer, 1999.

[4] H.D. Ellis, J.W. Shepherd, G.M. Davies, Identification of familiar and unfamiliar faces from internal and external features: some implications for theories of face recognition, Perception 8 (1979) 431-439.

[5] S.J. McKelvie, The role of eyes and mouth in the memory of a face, The American Journal of Psychology (1976) 311-323.

[6] N.D. Haig, Exploring recognition with interchanged facial features, Perception 15 (1986) 235-247.

[7] N.D. Haig, The effect of feature displacement on face recognition, Perception 42 (1984) 1158-1165.

[8] C.N. Stephan, P.L. Davidson, The placement of the human eyeball and canthi in craniofacial identification, Journal of Forensic Sciences 53 (2008) 612-619.

[9] S.E. Whitnall, The anatomy of the human orbit and accessory organs of vision, Oxford Medical Publications, London, 1921.

[10] W.W. Goldnamer, The anatomy of the human eye and orbit, Professional Press, 1923. [11] S.E. Whitnall, The anatomy of the human orbit and accessory organs of vision, Oxford University Press, London, 1932.

[12] E. Wolff, The anatomy of the eye and orbit, including the central connections, development, and comparative anatomy of the visual apparatus, H.K. Lewis, London, 1933. [13] A. Bron, R. Tripathi, B. Tripathi, Wolff's Anatomy of the Eye and Orbit. 8th, London, UK: Chapman \& Hall Medical, 1997. 
[14] C.N. Stephan, A.J.R. Haung, P.L. Davidson, Further evidence on the anatomical placement of the human eyeball for facial approximation and craniofacial superimposition, Journal of Forensic Sciences 54 (2009) 267-269.

[15] P. Guyomarc'h, B. Dutailly, C. Couture, H. Coqueugniot, Anatomical placement of the human eyeball in the orbit - validation using CT scans of living adults and prediction for facial approximation, Journal of forensic sciences 57 (2012) 1271-1275.

[16] S.-R. Kim, K.-M. Lee, J.-H. Cho, H.-S. Hwang, Three-dimensional prediction of the human eyeball and canthi for craniofacial reconstruction using cone-beam computed tomography, Forensic Science International 261 (2016) 164.e1-164.e8.

[17] T. Stewart, The points of attachment of the palpebral ligaments: their use in facial reconstructions on the skull, Journal of Forensic Science 28 (1983) 858-863.

[18] I. Bekerman, P. Gottlieb, M. Vaiman, Variations in Eyeball Diameters of the Healthy Adults, Journal of Ophthalmology 2014 (2014) 5.

[19] mediLexicon, Equator of Eyeball, mediLexicon, 2017.

[20] MeVisLab. <https://www. mevislab.de>, MeVis Medical Solutions AG, Germany, 2016.

[21] P.Z. Mala, Pronasale Position: An Appraisal of Two Recently Proposed Methods for Predicting Nasal Projection in Facial Reconstruction, Journal of Forensic Sciences 58(4) (2013) 957-963.

[22] A.C. Oettlé, F.P. Demeter, E.N. L'abbé, Ancestral Variations in the Shape and Size of the Zygoma, The Anatomical Record 300 (2017) 196-208.

[23] T. Balueva, E. Veselovskaya, E. Kobyliansky, Craniofacial reconstruction by applying the ultrasound method in live human populations, International Journal of Anthropology 24 (2009) 87-111.

[24] L. Farkas, Anthropometry of the head \& face, 2nd ed., Raven Press, New York, 1994.

[25] C.M. Özer, I.I. Öz, I. Serifoglu, M.Ç. Büyükuysal, Ç. Barut, Evaluation of Eyeball and Orbit in Relation to Gender and Age, Journal of Craniofacial Surgery 27 (2016) e793-e800.

[26] C.M. Wilkinson, S.A. Mautner, Measurement of eyeball protrusion and its application in facial reconstruction, Journal of Forensic Sciences 48 (2003) 12-16. 